Paper $36 \quad \begin{aligned} & \text { CCivil-Comp Press, 2012 } \\ & \text { Proceedings of the Eighth International Conference } \\ & \text { on Engineering Computational Technology, } \\ & \text { B.H.V. Topping, (Editor), } \\ & \text { Civil-Comp Press, Stirlingshire, Scotland }\end{aligned}$

\title{
Untangling and Smoothing of Quadrilateral and Hexahedral Meshes
}

\author{
T.J. Wilson ${ }^{1}$, J. Sarrate ${ }^{1}$, X. Roca ${ }^{1}$, R. Montenegro ${ }^{2}$ and J.M. Escobar ${ }^{2}$ \\ ${ }^{1}$ Laboratori de Calcul Numeric (LaCaN) \\ Universitat Politecnica de Catalunya, Barcelona, Spain \\ ${ }^{2}$ Instituto Universitario de Sistemas Inteligentes y Aplicaciones Numéricas en \\ Ingeniería (SIANI), Universidad de Las Palmas de Gran Canaria, Spain
}

\begin{abstract}
In this paper, we extend a simultaneous untangling and smoothing technique previously developed for triangular and tetrahedral meshes to quadrilateral and hexahedral ones. Specifically, we present a technique that iteratively untangles and smooths a given quadrilateral or hexahedral mesh by minimizing an objective function defined in terms of a modification of an algebraic quality measure. The proposed method optimizes the mesh quality by a local node relocation process. That is, without modifying the mesh connectivity. Finally, we present several examples to show that the proposed technique obtains valid meshes composed by high-quality quadrilaterals and hexahedra, even when we start from tangled meshes.
\end{abstract}

Keywords: mesh generation, hexahedral mesh, mesh smoothing, mesh untangling, mesh optimization.

\section{Introduction}

The accuracy and performance of a finite element analysis depend on the quality of the mesh on which the spatial domain has been discretized. Most three-dimensional meshes are composed of tetrahedral elements as they can be automatically generated to discretize an arbitrary domain by rapid and mature algorithms. While more challenging to mesh, hexahedral elements have several advantages over tetrahedral meshes both in structural and in fluid mechanics. Unfortunately, ready access to these advantages is hampered by the significant challenges regarding the generation of the hexahedral mesh. Several algorithms have been developed to generate hexahedral meshes. On the one hand, specific algorithms have been developed for specific types of geometries. For instance, the submapping method $[1,2]$ is devoted to blocky geometries or the sweep method $[3,4,5,6]$ is designed for extrusion geometries. On the other hand, 
several methods have been developed to mesh any geometry with an unstructured hexahedral mesh. For instance, 1 . dual methods such as wisker weaving [7] or the local dual contributions [8];2. primal methods that directly generates the hexahedral mesh such as grid based methods $[9,10,11]$, advancing front methods $[12,13]$, or the receding front method [14], and 3. primal methods that transform a tetrahedral mesh into a hexahedral mesh $[15,16]$. However, fully automatic unstructured hexahedral mesh generation algorithms are still not available, and at this stage, no automated technique exists which compares to tetrahedral methods such as Delaunay or advancing front techniques. Moreover, hexahedral meshes generated automatically can include poorly formed or even inverted elements that can affect the accuracy and invalidate any subsequent analysis. Therefore, it is of the major importance to develop an algorithm that can improve (untangle and smooth) the quality of a given hexahedral mesh.

Mesh smoothing is the key for keeping mesh shape regularity and avoiding a costly remeshing process. Several smoothing algorithms have been developed during the last decades to improve the quality of a given mesh. Most of them are based on geometrical or numerical reasoning $[17,18]$. Although they are fast from the computational point of view, they have some drawbacks. For instance, they can move nodes outside of a domain near non-convex boundaries. In addition, they are not designed to optimize any quality measure. To overcome these drawbacks, Knupp [19, 20, 21, 22] introduced a family of quality measures placed within an algebraic framework that have been intensively used during the last decade. These quality measures facilitate the development of smoothing methods based on its optimization. In fact, this optimization is transformed into a continuous minimization problem [19, 20, 23, 24].

The minimization process can be performed globally [24] or based on a local approach [25]. In short, these techniques consist on finding the new location that the mesh nodes must hold, in such a way that they optimize an objective function. This objective function is based on a measure of the quality of the local submesh formed by the set of elements connected to the free node. After repeating this process several times for all the nodes of the current mesh, quite satisfactory results can be achieved. Usually, objective functions are appropriate to improve the quality of a valid mesh, but they do not work properly when there are inverted elements. This is because they present singularities (barriers) when any element of the local submesh changes the sign of its Jacobian. In general, this procedure involves two steps [24, 25]: the first is for mesh untangling and the second one for mesh smoothing. Each step leads to a different objective function.

In this paper, we apply to quadrilateral and hexahedral meshes the shape distortion measure introduced by Knupp [22, 24]. The quality of the quadrilateral and hexahedral elements is defined in terms of their decomposition in triangular and tetrahedral subelements, respectively, Thus, the optimization methods for quadrilateral and hexahedral meshes inherit the properties of the methods for triangular and tetrahedral meshes. To avoid the need of defining two objectives functions for the untangling and smoothing process, we use the improvement proposed by Escobar et al. [26, 27, 28] for tetrahedral mesh optimization, where a simultaneous untangling and smoothing 
guided by the same objective function is introduced. This technique has been considered in different finite element applications. For example, it has been the key of the meccano method [29] and its recent application for isogeometric solid modeling [30]. Specifically, we present a technique that iteratively untangle and smooth a given quadrilateral or hexahedral mesh by minimizing an objective function defined in terms of an algebraic quality measure. It is important to point out that the proposed method optimizes the quality of quadrilateral and hexahedral meshes by a local node relocation process. That is, without modifying the mesh connectivity.

The paper is organized as follows. In the next section we review the concept of an algebraic shape quality metric for triangular and tetrahedral elements, based on the consideration of a target element. Section 3 summarizes the simultaneous untangling and smoothing of tetrahedral meshes. Then, in Section 4 we extend to quadrilateral and hexahedral elements the distortion and quality measures previously reviewed. The simultaneous untangling and smoothing technique for quadrilateral and hexahedral meshes is detailed in Section 5. We present several examples in Section 6 illustrating that the proposed technique can be used to obtain valid meshes (untangled) composed by high-quality (smoothed) quadrilaterals and hexahedra. Finally, several conclusions and future works are summarized in Section 7.

\section{Algebraic quality measures for triangles and tetra- hedra}

Knupp [21], introduced a family of distortion and quality measures placed within an algebraic framework. In this framework, the distortion measure of a simplex (triangle or tetrahedron) is measured in terms of the deviation from an ideal simplex, the element that represents the desired shape to achieve. In this Section, we first summarize the formulation of the algebraic framework for triangles. Second, we generalize it for tetrahedral elements.

Let $t$ denote a triangle in the physical space, and $t_{I}$ denote the ideal triangle. According to [21], to measure the deviation of the physical triangle from the ideal one, we want to determine the affine mapping (see Figure 1):

$$
\begin{aligned}
\mathbf{f}_{\mathbf{S}}: t_{I} & \longrightarrow t \\
\tilde{\mathbf{x}} & \longmapsto \mathbf{x}=\mathbf{S} \tilde{\mathbf{x}}+\mathbf{v},
\end{aligned}
$$

where $\tilde{\mathbf{x}}$ are the coordinates of the ideal element.

To this end, we consider a reference triangle, $t_{R}$, delimited by vertices $\mathbf{u}_{0}=(0,0)$, $\mathbf{u}_{1}(1,0)$ and $\mathbf{u}_{2}=(0,1)$ in the parametric space. If the ideal element, $t_{I}$, is delimited by the vertices: $\tilde{\mathbf{x}}_{0}=\left(\tilde{x}_{0}, \tilde{y}_{0}\right), \tilde{\mathbf{x}}_{1}=\left(\tilde{x}_{1}, \tilde{y}_{1}\right)$, and $\tilde{\mathbf{x}}_{2}=\left(\tilde{x}_{2}, \tilde{y}_{2}\right)$, the affine mapping that maps the reference element to the ideal one can be written as (see Figure 1):

$$
\begin{aligned}
\mathbf{f}_{\mathbf{W}}: t_{R} & \longrightarrow t_{I} \\
\mathbf{u} & \longmapsto \tilde{\mathbf{x}}=\mathbf{W u}+\tilde{\mathbf{x}}_{0},
\end{aligned}
$$


where

$$
\mathbf{W}=\left(\begin{array}{cc}
\tilde{x}_{1}-\tilde{x}_{0} & \tilde{x}_{2}-\tilde{x}_{0} \\
\tilde{y}_{1}-\tilde{y}_{0} & \tilde{y}_{2}-\tilde{y}_{0}
\end{array}\right)
$$

Similarly, if the triangle in the physical space is delimited by nodes $\mathbf{x}_{0}=\left(x_{0}, y_{0}\right)$, $\mathbf{x}_{1}=\left(x_{1}, y_{1}\right)$ and $\mathbf{x}_{2}=\left(x_{2}, y_{2}\right)$, the affine mapping that maps the reference element, $t_{R}$, into the physical triangle, $t$, can be written as (see Figure 1):

$$
\begin{aligned}
\mathbf{f}_{\mathbf{A}}: t_{R} & \longrightarrow t \\
\mathbf{u} & \longmapsto \mathbf{x}=\mathbf{A} \mathbf{u}+\mathbf{x}_{0},
\end{aligned}
$$

where

$$
\mathbf{A}=\left(\begin{array}{cc}
x_{1}-x_{0} & x_{2}-x_{0} \\
y_{1}-y_{0} & y_{2}-y_{0}
\end{array}\right)
$$

The desired affine mapping, Equation (1), that maps the ideal triangle to the physical triangle can be determined by composing the inverse of $f_{\mathrm{W}}$, Equation (3), with function $f_{A}$, Equation (5):

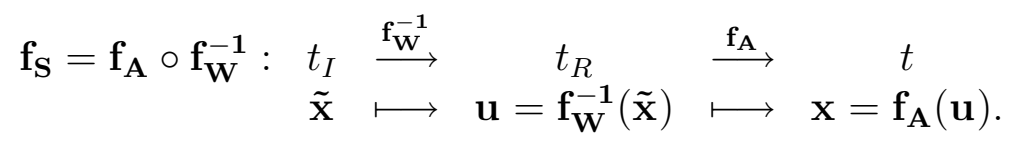

Therefore, the analytical expression of affine mapping $\mathbf{f}_{\mathrm{S}}$ is

$$
\begin{aligned}
\mathbf{x} & =\mathbf{f}_{\mathbf{S}}(\tilde{\mathbf{x}})=\mathbf{f}_{\mathbf{A}}\left(\mathbf{f}_{\mathbf{W}}^{-1}(\tilde{\mathbf{x}})\right)=\mathbf{A f}_{\mathbf{W}}^{-\mathbf{1}}(\tilde{\mathbf{x}})+\mathbf{x}_{0}=\mathbf{A}\left(\mathbf{W}^{-1} \tilde{\mathbf{x}}+\tilde{\mathbf{v}}\right)+\mathbf{x}_{0} \\
& =\mathbf{A W}^{-1} \tilde{\mathbf{x}}+\mathbf{A} \tilde{\mathbf{v}}+\mathbf{x}_{0},
\end{aligned}
$$

being $\tilde{\mathbf{v}}=-\mathbf{W}^{-1} \tilde{\mathbf{x}}_{0}$. Comparing this expression with Equation (1) we realize that:

$$
\begin{aligned}
& \mathbf{S}=\mathbf{A} \mathbf{W}^{-\mathbf{1}} \\
& \mathbf{v}=\mathbf{A} \tilde{\mathbf{v}}+\mathbf{x}_{0} .
\end{aligned}
$$

Note that if we consider $\tilde{\mathbf{x}}_{0}=(0,0)$, then $\tilde{\mathbf{v}}=(0,0)$ and consequently, from Equation (9), $\mathbf{v}=\mathbf{x}_{0}$. The Jacobian matrix of the affine mapping (1), $\mathbf{S}$, is called weighted Jacobian matrix in reference [21].

Note that using this formulation, we can select different ideal elements to generate elements with different geometric properties. For isotropic triangular meshes, the ideal element is selected as the equilateral triangle. Thus, we have

$$
\mathbf{W}=\left[\begin{array}{cc}
1 & 1 / 2 \\
0 & \sqrt{3} / 2
\end{array}\right]
$$

Similar reasoning can be developed for tetrahedral elements. In this case, if the tetrahedron in the physical space is delimited by the vertices: $\mathbf{x}_{0}=\left(x_{0}, y_{0}, z_{0}\right)$, $\mathbf{x}_{1}=\left(x_{1}, y_{1}, z_{1}\right), \mathbf{x}_{2}=\left(x_{2}, y_{2}, z_{2}\right)$, and $\mathbf{x}_{3}=\left(x_{3}, y_{3}, z_{3}\right)$, then we have:

$$
\mathbf{A}=\left(\begin{array}{ccc}
x_{1}-x_{0} & x_{2}-x_{0} & x_{3}-x_{0} \\
y_{1}-y_{0} & y_{2}-y_{0} & y_{3}-y_{0} \\
z_{1}-z_{0} & z_{2}-z_{0} & z_{3}-z_{0}
\end{array}\right)
$$




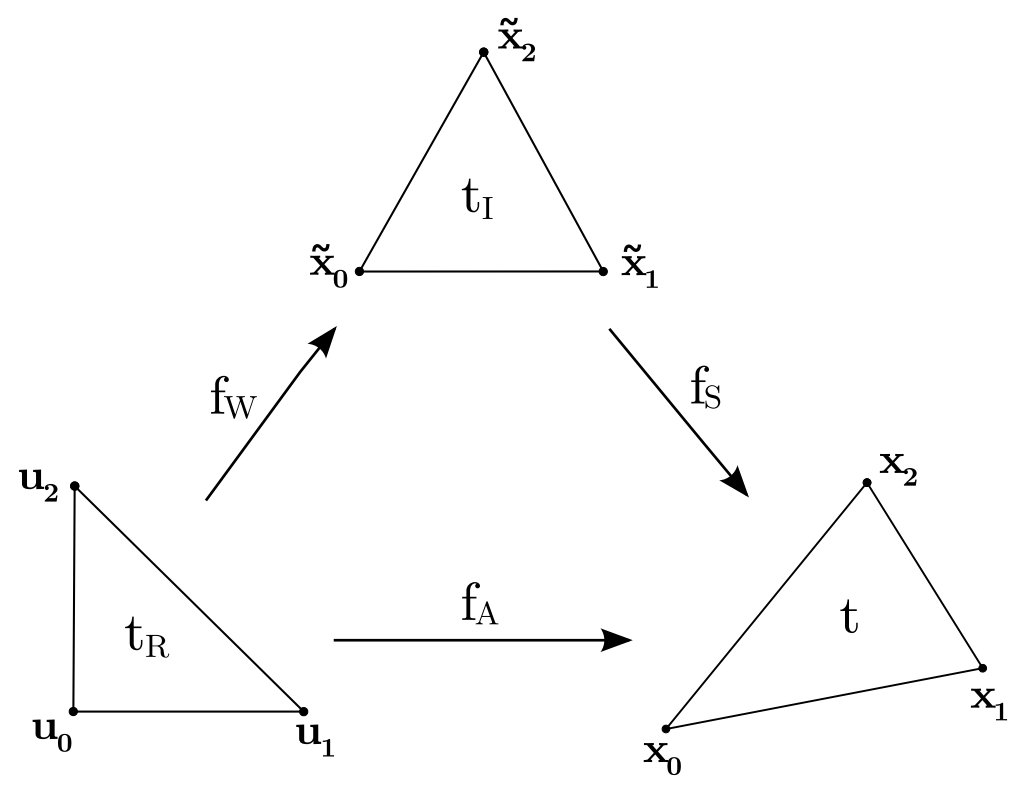

Figure 1: Affine mappings for triangular elements

In addition, for isotropic tetrahedral meshes, the ideal element is defined as an equilateral tetrahedron. Thus, we have

$$
\mathbf{W}=\left[\begin{array}{ccc}
1 & 1 / 2 & 1 / 2 \\
0 & \sqrt{3} / 2 & \sqrt{3} / 6 \\
0 & 0 & \sqrt{2 / 3}
\end{array}\right]
$$

The weighted Jacobian matrix $\mathbf{S}$ defined in (8) contains information about how much we have "distorted" the ideal element to become the physical one. Based on this matrix, Knupp [21] introduced the shape distortion measure

$$
\eta(\mathbf{S})=\frac{|\mathbf{S}|^{2}}{n \sigma(\mathbf{S})^{2 / n}}
$$

where $\sigma(\mathbf{S})=\operatorname{det}(\mathbf{S})$ is the determinant of $\mathbf{S},|\mathbf{S}|=\sqrt{\operatorname{tr}\left(\mathbf{S}^{\mathbf{T}} \mathbf{S}\right)}$ is its Frobenius norm, and $n$ is the space dimension ( $n=2$ for triangles and $n=3$ for tetrahedra). If the physical element is not inverted the image of this function is the interval $[1, \infty]$, achieving $\infty$ only when the physical element is degenerated, and 1 when it becomes the ideal triangle.

The shape quality measure is defined as the inverse of the shape distortion measure (see [21])

$$
q(\mathbf{S})=\frac{1}{\eta(\mathbf{S})}=\frac{n \sigma(\mathbf{S})^{2 / n}}{|\mathbf{S}|^{2}}
$$

Note that the shape quality measure reaches a maximum value of 1 for the ideal element, and a minimum value 0 for degenerated elements. 


\section{Simultaneous untangling and smoothing of triangu- lar and tetrahedral meshes}

Given a triangular or a tetrahedral mesh $\mathcal{M}$, let $\mathcal{V}$ be the set of inner nodes, and let $v$ be a given node $v \in \mathcal{V}$. We define the local submesh associated to $v, \mathcal{M}_{v}$, as the set of elements that contain node $v$. The simultaneous untangling and smoothing algorithm for triangular [27] and tetrahedral [26] meshes is based on the optimization of an objective function defined in terms of the distortion measure (10) of the elements in the local submesh .

Let $\mathbf{x}$ be the coordinates of the inner node $v$. Assume that the local submesh, $\mathcal{M}_{v}$, is composed by $M$ elements (triangles or tetrahedra in our case). Let $\mathbf{S}_{k}$ be the weighted Jacobian matrix of the $k$ th element of $\mathcal{M}_{v}$. Then, according to [24], the objective function to minimize in the $k$ th element is its shape distortion measure:

$$
\eta_{k}(\mathbf{x})=\frac{\left|\mathbf{S}_{k}(\mathbf{x})\right|^{2}}{n \sigma\left(\mathbf{S}_{k}(\mathbf{x})\right)^{2 / n}} .
$$

We define the objective function on node $v$ as the p-norm of the objective function of all the elements of its local submesh:

$$
K_{\eta}(\mathbf{x})=\left(\sum_{k=1}^{M}\left(\eta_{k}\right)^{p}(\mathbf{x})\right)^{1 / p} .
$$

In this work, we use $p=2$. We define the feasible region as the set of points where the free node, $v$, can be located to get a valid mesh. Concretely, the feasible region is the interior of the polygonal set $\mathcal{H}=\cap_{k=1}^{k=M} H_{k}$ where $H_{k}$ are the half-planes defined by $\sigma_{k}(\mathbf{x}) \geq 0$. We say that an element of the mesh is tangled if $\sigma(\mathbf{x})<0$, and degenerated if $\sigma(\mathbf{x})=0$.

Note that the objective function (13) presents several asymptotes (or barriers) at the boundary of the feasible region, where $\sigma=0$. In addition, the objective function also presents local minima outside the feasible region. Then, the optimization algorithm, that is indeed a minimization, can lead to a local minimum that is not the optimal position. Moreover, this local minimum may correspond to a tangled configuration.

To overcome this drawback, Escobar et al. [26, 27] modified the objective function (13) in order to obtain a new objective function without asymptotes and with a global minimum near the optimal position of the original objective function (13). Specifically, they replace $\sigma$ in (12) by

$$
h(\sigma)=\frac{1}{2}\left(\sigma+\sqrt{\sigma^{2}+4 \delta^{2}}\right),
$$

where $\delta$ is an arbitrary small parameter that is chosen depending on the problem (see $[27,26,31,32]$ for further details). Taking into account this modification, see Equation (14), the new objective function for the local mesh is

$$
K_{\eta}^{*}(\mathbf{x})=\left(\sum_{k=1}^{M}\left(\eta_{k}^{*}\right)^{p}(\mathbf{x})\right)^{1 / p}
$$


where

$$
\eta_{k}^{*}=\frac{\left|\mathbf{S}_{k}\right|^{2}}{n h\left(\sigma_{k}\right)^{2 / n}}
$$

\section{Distortion and quality measures for quadrilateral and hexahedral elements}

According to Knupp [24], the distortion measure of a quadrilateral element can be computed by decomposing it into four triangles, see Figure 2. Similarly, the distortion measure of an hexahedral element can be computed by decomposing it into eight tetrahedra, see Figure 3. Thus, given a quadrilateral (hexahedral) element, if we denote the shape distortion measure of the $i$ th triangle (tetrahedron) by $\eta\left(\mathbf{S}_{i}\right)$, the shape distortion measure of a quadrilateral (hexahedral) elements is:

$$
\eta=\frac{1}{N} \sum_{i=1}^{N} \eta\left(\mathbf{S}_{i}\right)=\frac{1}{N} \sum_{i=1}^{N} \frac{\left|\mathbf{S}_{i}\right|^{2}}{n \sigma\left(\mathbf{S}_{i}\right)^{2 / n}},
$$

where $N$ is the number of nodes of the quadrilateral (hexahedral) element.
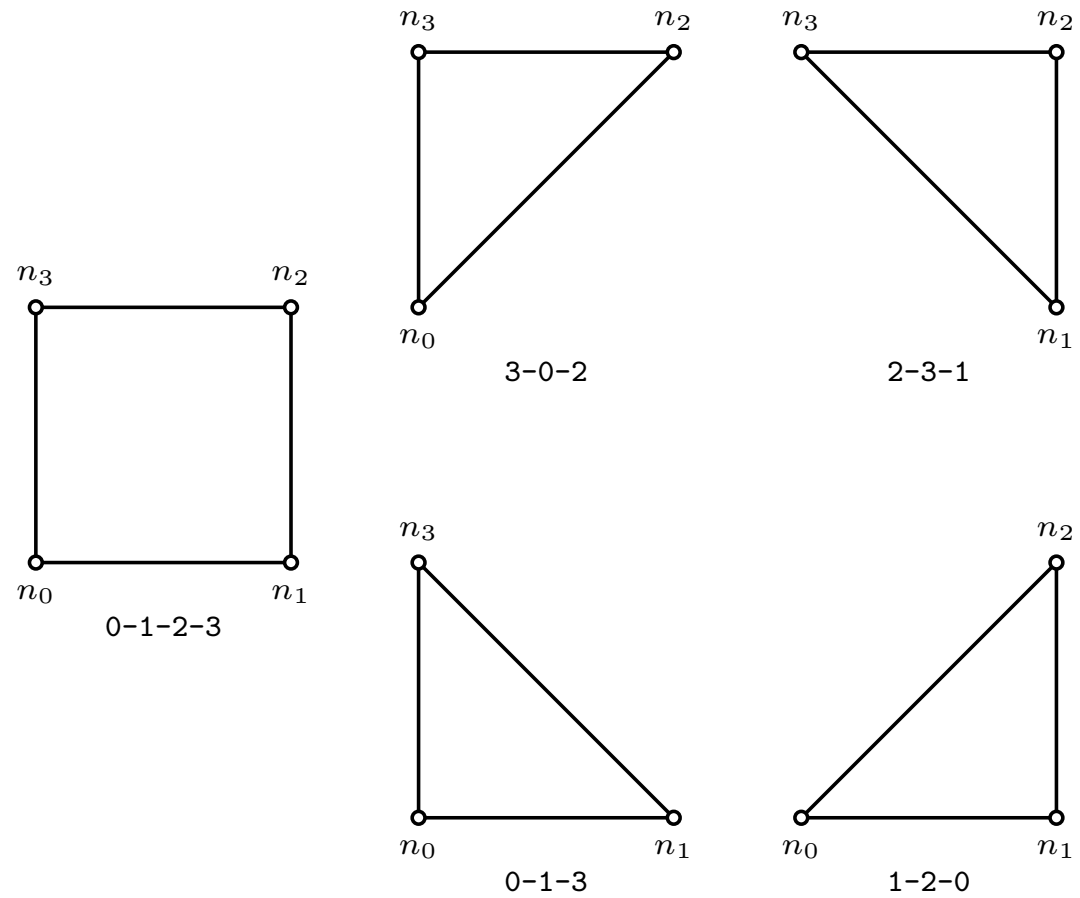

Figure 2: Decomposition of a quadrilateral into four triangular sub-elements 

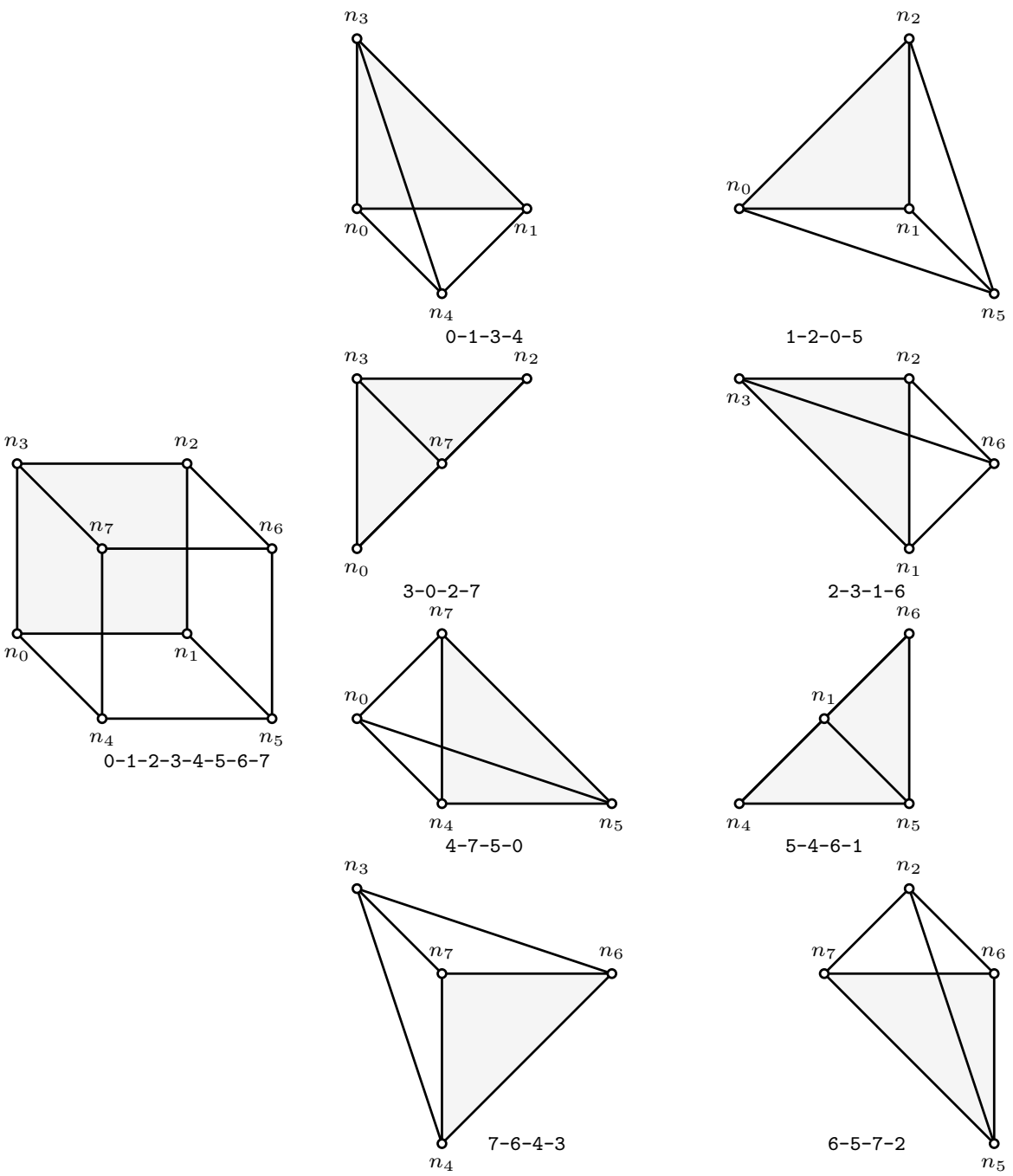

Figure 3: Decomposition of a hexahedron into eight tetrahedral sub-elements

The ideal quadrilateral element is a square. Thus, if it is subdivided into four triangles, we get that the ideal triangle for this case is rectangle and isosceles. Similarly, the ideal hexahedral element is a cube. Hence, if it is subdivided into eight tetrahedra, we get that the ideal tetrahedron is the rectangle and isosceles tetrahedron. Therefore, for each sub-triangle of a quadrilateral element, and for each sub-tetrahedron of a hexahedral element we get that the mapping between the reference and the ideal element, see (3), is given by

$$
\mathbf{W}=\left[\begin{array}{ll}
1 & 0 \\
0 & 1
\end{array}\right] \quad \mathbf{W}=\left[\begin{array}{lll}
1 & 0 & 0 \\
0 & 1 & 0 \\
0 & 0 & 1
\end{array}\right]
$$

respectively. 
Using Equation (17), and according to Equation (11), the shape quality measure for quadrilateral (hexahedral) elements is defined as:

$$
q=\frac{1}{\eta}=\frac{1}{\frac{1}{N} \sum_{i=1}^{N} \eta\left(\mathbf{S}_{i}\right)} .
$$

\section{Simultaneous untangling and smoothing of quadri- lateral and hexahedral meshes}

According to the notation introduced in the previous two sections, given a quadrilateral (hexahedral) mesh, we consider an inner node $v \in \mathcal{V}$ and its local submesh, $\mathcal{M}_{v}$. Let $\mathrm{x}$ be the coordinates of the node $v$, and $M$ the number of quadrilateral (hexahedral) elements in $\mathcal{M}_{v}$. Analogously to the triangular (tetrahedral) case, we define the objective function on a free node $v$ as the p-norm of the objective function of all the quadrilaterals (hexahedral) elements of its local submesh, see Equation (13). However, for quadrilateral (hexahedral) elements the distortion measure is computed according to Equation (17).

For quadrilateral and hexahedral elements, the objective function (13) also presents asymptotes (or barriers) and local minima that prevent its use in a continuous minimization procedure. Therefore, the modification introduced in Equation (14) is also used when the distortion measure of each sub-element is computed in Equation (17). Thus, the modified distortion measures for quadrilateral (hexahedral) elements is:

$$
K_{\eta}^{*}(\mathbf{x})=\left(\sum_{k=1}^{M}\left(\eta_{k}^{*}\right)^{p}(\mathbf{x})\right)^{1 / p}
$$

where

$$
\eta_{k}^{*}(\mathbf{x})=\frac{1}{N} \sum_{i=1}^{N} \eta^{*}\left(\mathbf{S}_{i}(\mathbf{x})\right),
$$

being

$$
\eta^{*}\left(\mathbf{S}_{i}(\mathbf{x})\right)=\frac{\left.\mid \mathbf{S}_{i}(\mathbf{x})\right)\left.\right|^{2}}{n h\left(\sigma_{i}\right)^{2 / n}}
$$

Similar to the methods proposed by $[24,26]$ we propose a continuous optimization procedure to simultaneously untangle and smooth quadrilateral and hexahedral meshes. Specifically, for each free node we propose to minimize the shape distortion measure of its local submesh (19). This way, we are maximizing the shape quality measure since the quality measure is the inverse of the distortion measure, see Equation (11) or (18).

To minimize the objective function (19) we perform a local non-linear minimization with updates. This approach is similar to the Gauss-Seidel technique for solving 
linear systems. Specifically, we loop on all the nodes that are not located on the boundary of the mesh. Then, in each step we move the position of one node, and we fix the other ones. To this end, we minimize the local objective function of the node and we update the node location to the optimum. Then, we repeat the process again for the next node.

\section{Examples}

In order to illustrate the behavior of the proposed mesh optimization method, we present four examples in this section. In all cases, the method is capable to untangle the initial mesh, increasing the values of the minimum quality, $\min (q)$, and of the mean value of the quality, $\bar{q}$. In addition, the standard deviation of the quality of the elements, $\sigma(q)$, is also reduced.

In the first example we apply the developed method to a structured quadrilateral mesh on a convex domain, see Figure 4 . The coordinates of inner nodes are randomly perturbed and all inner nodes are placed outside of the domain. Thus, the input mesh is composed by tangled elements, see Figure 4(a). The smoothed mesh is shown in 4(b). As expected, the optimized mesh is only composed by square elements. Table 1 summarizes the quality statistics for this example.

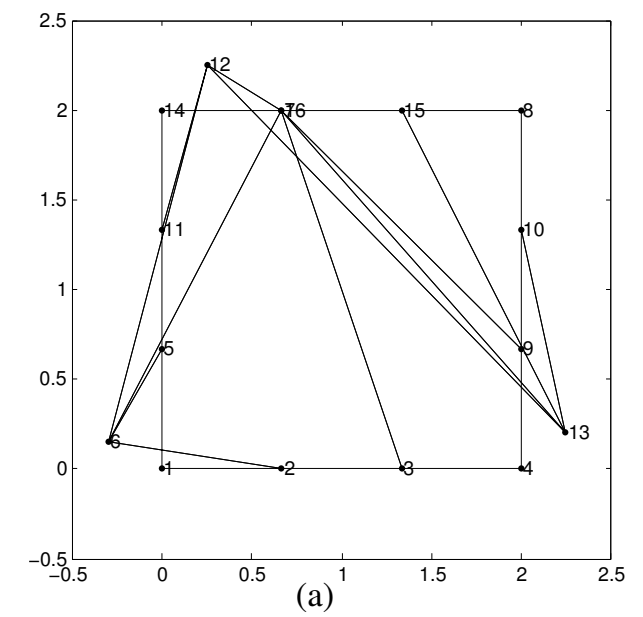

Figure 4: Smoothing of a quadrilateral mesh: (a) tangled mesh, and (b) optimized mesh.

The second example illustrates the behavior of the developed method when it is applied to a non-convex domain. Figure 5(a) shows one fourth of a ring with outer radius $r_{\text {out }}=10$ and inner radius $r_{\text {in }}=3$, meshed with quadrilateral elements. The interior elements were arranged in a square domain, decreasing the quality of the elements and in some cases, tangling them. The smoothed mesh is shown in 5(b). Table 2 summarizes the quality statistics for the presented meshes. Note that after smoothing, no element appears with shape quality measure lower than 0.69 . 


\begin{tabular}{lcc}
\hline & Initial mesh & Optimized mesh \\
\hline $\min (q)$ & 0.000 & 1.000 \\
$\max (q)$ & 0.444 & 1.000 \\
$\bar{q}$ & 0.065 & 1.000 \\
$\sigma(q)$ & 0.141 & 0.000 \\
\hline
\end{tabular}

Table 1: Quality statistics of the meshes corresponding to the square domain.
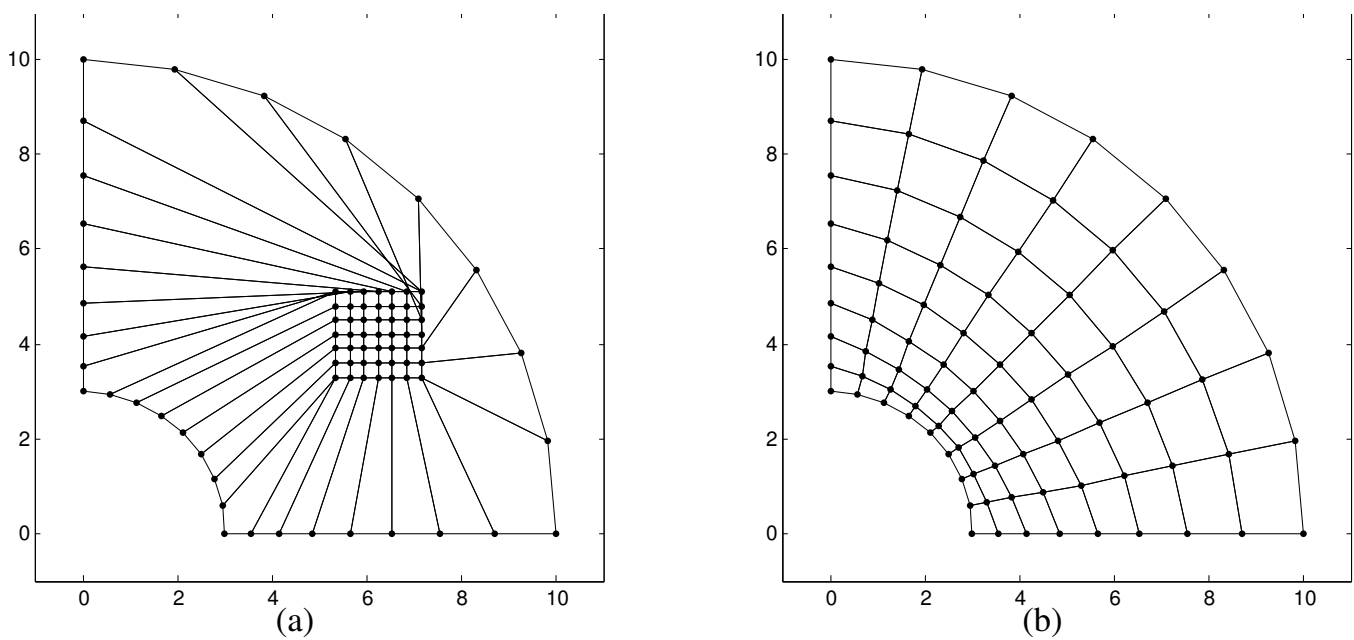

Figure 5: Smoothing of a mesh on a ring-shaped domain: (a) tangled mesh, and (b) optimized mesh.

\begin{tabular}{lcc}
\hline & Initial mesh & Optimized mesh \\
\hline $\min (q)$ & 0.000 & 0.690 \\
$\max (q)$ & 1.000 & 0.967 \\
$\bar{q}$ & 0.612 & 0.902 \\
$\sigma(q)$ & 0.445 & 0.079 \\
\hline
\end{tabular}

Table 2: Quality statistics of the meshes corresponding to the ring-shaped domain.

The third example is devoted to the optimization of a hexahedral mesh. Figure 6(a) shows a cylinder with radius $r=5$ and height $z=5$ with non-planar cap surfaces. It is important to point out that we have created several flat elements (with zero volume) attached to both cap surfaces. After applying the proposed untangling and smoothing method, see Figure 6(b) we obtain a mesh composed by valid elements. Table 3 summarizes the statistics of both meshes. Note that high-quality hexahedral elements are obtained near the cap surfaces.

The fourth example shows an application of the proposed optimization method to the receding front method [14]. Using this method we generate an hexahedral mesh around an airplane. To test the capabilities of the optimization method we do 

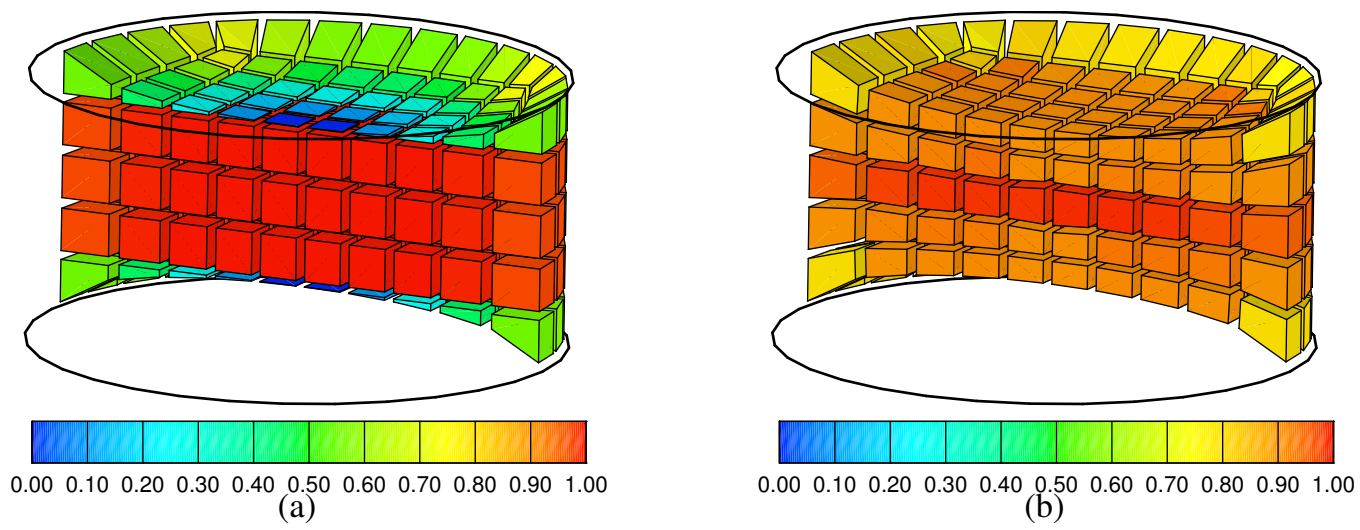

Figure 6: Smoothing of a mesh of a cylinder: (a) tangled mesh, and (b) optimized mesh. The color map represents the element shape quality.

\begin{tabular}{lcc}
\hline & Initial mesh & Optimized mesh \\
\hline $\min (q)$ & 0.000 & 0.768 \\
$\max (q)$ & 0.994 & 0.971 \\
$\bar{q}$ & 0.749 & 0.907 \\
$\sigma(q)$ & 0.286 & 0.057 \\
\hline
\end{tabular}

Table 3: Quality statistics of the meshes corresponding to the cylinder.

not smooth the initial mesh during the generation process. Thus, it contains invalid elements. Figure 7(a) presents a cross-section of an initial hexahedral mesh around the airplane. Note that invalid elements appear. Figure 7(b) shows the optimized mesh. It is important to point out that the final mesh is composed by high-quality elements. Table 4 summarizes the statistics of both meshes.

\begin{tabular}{lcc}
\hline & Initial mesh & Optimized mesh \\
\hline $\min (q)$ & 0.094 & 0.605 \\
$\max (q)$ & 0.997 & 0.997 \\
$\bar{q}$ & 0.816 & 0.913 \\
$\sigma(q)$ & 0.127 & 0.064 \\
\hline
\end{tabular}

Table 4: Quality statistics of the meshes corresponding to the airplane.

\section{Concluding remarks}

In this paper, we have presented a new method to simultaneously untangle and smooth quadrilateral and hexahedral meshes. To this end, we propose to use the standard definition of the shape quality measure for quadrilateral and hexahedral elements, and 


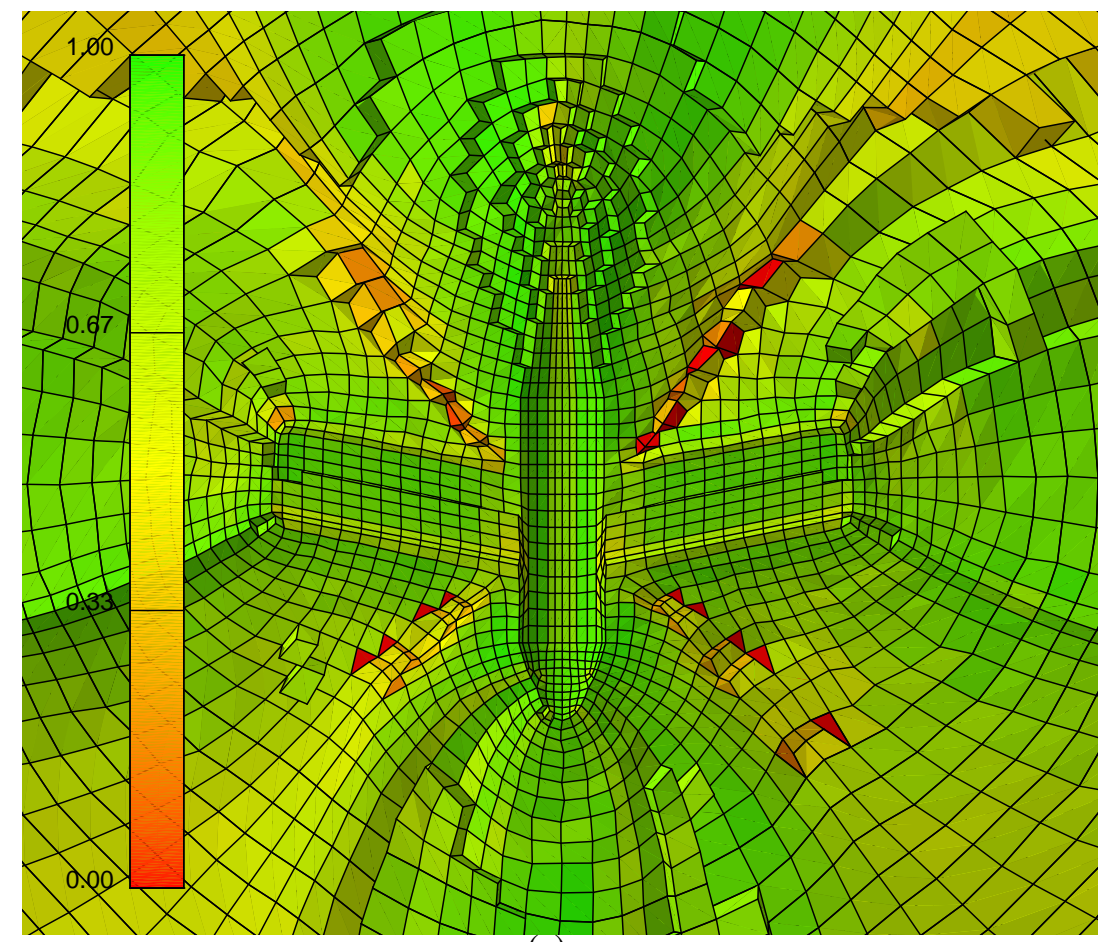

(a)

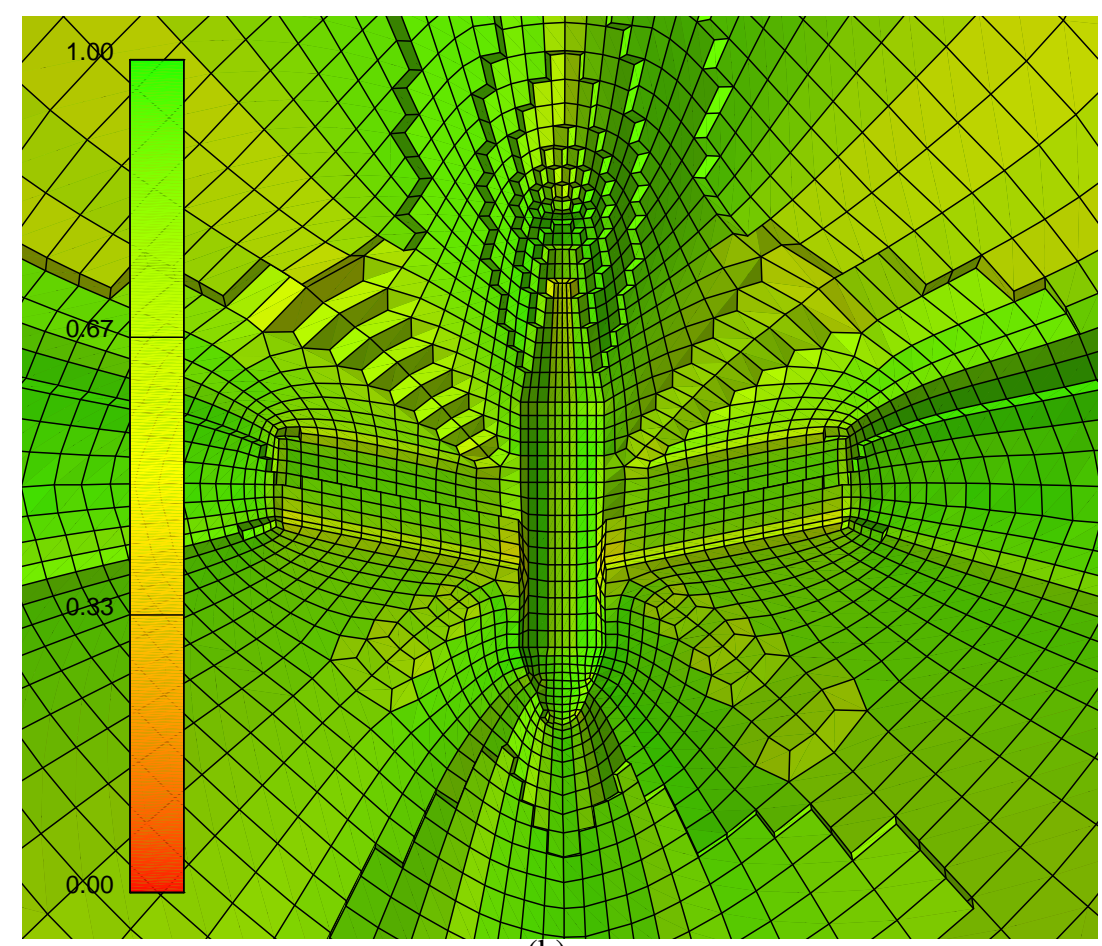

(b)

Figure 7: Smoothing of a hexahedral mesh around an airplane: (a) original mesh, and (b) optimized mesh. The color map represents the element shape quality. 
to minimize it using a continuous minimization procedure. To avoid the singularities (vertical asymptotes) of the objective we have modified it in such a way that the modified objective function is smooth all over $\mathbb{R}^{3}$ and has the global minimum near the global minimum of the original objective function. The regularity shown by the modified objective functions allows the use of standard optimization algorithms as steepest descent, conjugate gradient, Newton, etc. In principle, a similar modification could be also applicable to other objective functions having the same behavior (that are written in terms of $\sigma^{-1}$ ). Further research is needed to increase the computational efficiency of the developed optimization method. In particular, special attention will be focused in its implementation. Note that this technique can be implemented in a parallel algorithm. Thus, the implementation of the method in different parallel architectures will also be analyzed.

\section{Acknowledgements}

This work has been partially supported by the Spanish Government and FEDER under grant contracts: CGL2011-29396-C03-01 and DPI2011-23058.

\section{References}

[1] M. Whiteley, D.R. White, S. Benzley, T.D. Blacker, "Two and three-quarter dimensional meshing facilitators", Engineering with Computers, 12(3-4): 144154, 1996.

[2] E. Ruiz-Gironés, J. Sarrate, "Generation of structured hexahedral meshes in volumes with holes", Finite Elements in Analysis and Design, 46(10): 792 804, 2010.

[3] M.L. Staten, S.A. Canann, S.J. Owen, "BMSweep: locating interior nodes during sweeping", Engineering with Computers, 15: 212-218, 1999.

[4] D.R. White, S. Saigal, S.J. Owen, "CCSweep: an automatic decomposition of multi-sweep volumes”, Engineering with Computers, 20: 222-236, 2004.

[5] X. Roca, J. Sarrate, "An automatic and general least-squares projection procedure for sweep meshing”, Engineering with Computers, 26(4): 391-406, 2010.

[6] E. Ruiz-Gironés, X. Roca, J. Sarrate, "Using a computational domain and a three-stage node location procedure for multi-sweeping algorithms", Advances in Engineering Software, 42(9): 700 - 713, 2011.

[7] T.J. Tautges, T.D. Blacker, S.A. Mitchell, “The whisker weaving algorithm: a connectivity-based method for constructing all-hexahedral finite element meshes", International Journal for Numerical Methods in Engineering, 39(19): 3327-3350, 1996.

[8] X. Roca, J. Sarrate, "Local dual contributions: Representing dual surfaces for block meshing", International Journal for Numerical Methods in Engineering, 83(6): 709-740, 2010. 
[9] R. Schneiders, "A grid-based algorithm for the generation of hexahedral element meshes", Engineering with Computers, 12(3): 168-177, 1996.

[10] Y. Zhang, C. Bajaj, "Adaptive and quality quadrilateral/hexahedral meshing from volumetric data", Computer Methods in Applied Mechanics and Engineering, 195(9-12): 942-960, 2006.

[11] J. Qian, Y. Zhang, "Sharp Feature Preservation in Octree-Based Hexahedral Mesh Generation for CAD Assembly Models", in Proceedings of the 19th International Meshing Roundtable, pages 243-262. Springer, 2010.

[12] T.D. Blacker, R.J. Meyers, "Seams and wedges in Plastering: a 3-D hexahedral mesh generation algorithm", Engineering with computers, 9(2): 83-93, 1993.

[13] M.L. Staten, R.A. Kerr, S.J. Owen, T.D. Blacker, M. Stupazzini, K. Shimada, "Unconstrained plastering-Hexahedral mesh generation via advancing-front geometry decomposition", International Journal for Numerical Methods in Engineering, 81(2): 135-171, 2010.

[14] E. Ruiz-Gironés, X. Roca, J. Sarrate, "The receding front method applied to hexahedral mesh generation of exterior domains", Engineering with Computers, DOI: 10.1007/s00366-011-0233-y.

[15] S. Meshkat, D. Talmor, "Generating a mixed mesh of hexahedra, pentahedra and tetrahedra from an underlying tetrahedral mesh", International Journal for Numerical Methods in Engineering, 49(1-2): 17-30, 2000.

[16] S.J. Owen, S. Saigal, "H-Morph: an indirect approach to advancing front hex meshing", International Journal for Numerical Methods in Engineering, 49(12): 289-312, 2000.

[17] L. Herrmann, "Laplacian-isoparametric grid generation scheme", Journal of the Engineering Mechanics Division, 102(5): 749-756, 1976.

[18] S. Giuliani, "An algorithm for continuous rezoning of the hydrodynamic grid in arbitrary lagrangian-eulerian computer codes", Nuclear Engineering and Design, 72(2): 205-212, 1982.

[19] P.M. Knupp, "Achieving finite element mesh quality via optimization of the Jacobian matrix norm and associated quantities. Part I - A framework for surface mesh optimization", International Journal of Numerical Methods in Engineering, 48: 401-420, 2000.

[20] P.M. Knupp, "Achieving finite element mesh quality via optimization of the Jacobian matrix norm and associated quantities. Part II - A framework for volume mesh optimization and the condition number of the Jacobian matrix", International Journal of Numerical Methods in Engineering, 48: 1165-1185, 2000.

[21] P.M. Knupp, "Algebraic mesh quality metrics", SIAM Journal on Scientific Computing, 23(1): 193-218, 2001.

[22] P.M. Knupp, "Algebraic mesh quality metrics for unstructured initial meshes", Finite Elements in Analysis and Design, 39(3): 217-241, 2003.

[23] L. Freitag, P.M. Knupp, "Tetrahedral mesh improvement via optimization of the element condition number", International Journal of Numerical Methods in Engineering, 53: 1377-1391, 2002.

[24] P.M. Knupp, "A method for hexahedral mesh shape optimization", International 
Journal of Numerical Methods in Engineering, 58(2): 319-332, 2003.

[25] L.A. Freitag, P. Plassmann, "Local optimization-based simplicial mesh untangling and improvement", International Journal of Numerical Methods in Engineering, 49: 109-125, 2000.

[26] J.M. Escobar, E. Rodríguez, R. Montenegro, G. Montero, J.M. González-Yuste, "Simultaneous untangling and smoothing of tetrahedral meshes", Computer Methods in Applied Mechanics and Engineering, 192(25): 2775-2787, 2003.

[27] J. Escobar, G. Montero, R. Montenegro, E. Rodríguez, "An algebraic method for smoothing surface triangulations on a local parametric space", International Journal of Numerical Methods in Engineering, 66(4): 740-760, 2006.

[28] J.M. Escobar, E. Rodríguez, R. Montenegro, G. Montero, J.M. GonzálezYuste, "SUS code - Simultaneous mesh untangling and smoothing code", 2010, URL www.dca.iusiani.ulpgc.es/proyecto2008-2011/ html/Software.html.

[29] R. Montenegro, J.M. Cascón, J.M. Escobar, E. Rodríguez, G. Montero, “An automatic strategy for adaptive tetrahedral mesh generation", Applied Numerical Mathematics, 59: 2203-2217, 2009.

[30] J.M. Escobar, J.M. Cascón, E. Rodríguez, R. Montenegro, "A new approach to solid modeling with trivariate T-splines based on mesh optimization", Computer Methods in Applied Mechanics and Engineering, 200: 3210 - 3222, 2011.

[31] T.J. Wilson, "Simultaneous untangling and smoothing of thetrahedral meshes", Master's thesis, ETSE Camins Canals i Ports de Barcelona, Universitat Politècnica de Catalunya, June 2011.

[32] A. Gargallo-Peiró, "Smoothing and untangling of meshes on parameterized surfaces", Master's thesis, Facultat de Matemàtiques, Universitat Politècnica de Catalunya, February 2011. 